\title{
Image-based effective feature generation for Protein Structural Class and Ligand Binding prediction
}

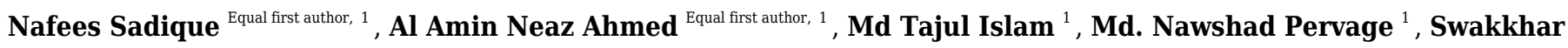 \\ Shatabda ${ }^{\text {Corresp. } 1}$ \\ 1 Department of Computer Science and Engineering, United International University, Dhaka, Dhaka, Bangladesh \\ Corresponding Author: Swakkhar Shatabda \\ Email address: swakkhar@cse.uiu.ac.bd
}

Proteins are the building blocks of all cells in both human and all our living creatures of the world. Most of the work in the living organism is performed by Proteins. Proteins are polymers of amino acid monomers which are biomolecules or macromolecules. The tertiary structure of protein represents the threedimensional shape of a protein. The functions, classification and binding sites are governed by protein's tertiary structure. If two protein structures are alike then the two proteins can be of the same kind implying similar structural class and ligand binding properties. In this paper, we have used protein tertiary structure to generate effective features for applications in structural similarity to detect structural class and ligand binding. Firstly, we analyze the effectiveness of a group of image-based features to predict the structural class of a protein. These features are derived from the image generated by the distance matrix of the tertiary structure of a given protein. They include local binary pattern histogram, Gabor filtered local binary pattern histogram, separate row multiplication matrix with uniform local binary pattern histogram, neighbor block subtraction matrix with uniform local binary pattern histogram and atom bond. Separate row multiplication matrix and neighbor block subtraction matrix filters as well as atom bond is our novels. The experiments were done on a standard benchmark dataset. We have demonstrated the effectiveness of these features over a large variety of supervised machine learning algorithms. Experiments suggest SVM is the best performing classifier on the selected dataset using the set of features. We believe the excellent performance of Hybrid LBP in terms of accuracy would motivate the researchers and practitioners to use it to identify protein structural class. To facilitate that, a classification model using Hybrid LBP is readily available for use at http://brl.uiu.ac.bd/PL/.

Protein-Ligand binding is accountable for managing the tasks of biological receptors that help to cure diseases and many more. So, binding prediction between protein and ligand is important for understanding a protein's activity or to accelerate docking computations in virtual screening-based drug design. Protein-Ligand Binding Prediction requires three-dimensional tertiary structure of the target protein to be searched for ligand binding. In this paper, we've proposed a supervised learning algorithm for predicting Protein-Ligand Binding which is a Similarity-Based Clustering approach using the same set of features. Our algorithm works better than the most popular and widely used machine learning algorithms. 


\title{
Image-based effective feature generation for Protein Structural Class and Ligand Binding prediction
}

\author{
Nafees Sadique ${ }^{1, *}$, Al Amin Neaz Ahmed ${ }^{1, *}$, Md Tajul Islam $^{1}$, Md. \\ Nawshad Pervage ${ }^{1}$, and Swakkhar Shatabda ${ }^{1}$
${ }^{1}$ Department of Computer Science and Engineering , United International University, Bangladesh* \\ Corresponding author: \\ Last Author ${ }^{1}$ \\ Email address: swakkhar@cse.uiu.ac.bd
}

\section{ABSTRACT}

Proteins are the building blocks of all cells in both human and all our living creatures of the world. Most of the work in the living organism is performed by Proteins. Proteins are polymers of amino acid monomers which are biomolecules or macromolecules. The tertiary structure of protein represents the three-dimensional shape of a protein. The functions, classification and binding sites are governed by protein's tertiary structure. If two protein structures are alike then the two proteins can be of the same kind implying similar structural class and ligand binding properties. In this paper, we have used protein tertiary structure to generate effective features for applications in structural similarity to detect structural class and ligand binding. Firstly, we analyze the effectiveness of a group of image-based features to predict the structural class of a protein. These features are derived from the image generated by the distance matrix of the tertiary structure of a given protein. They include local binary pattern histogram, Gabor filtered local binary pattern histogram, separate row multiplication matrix with uniform local binary pattern histogram, neighbor block subtraction matrix with uniform local binary pattern histogram and atom bond. Separate row multiplication matrix and neighbor block subtraction matrix filters as well as atom bond is our novels. The experiments were done on a standard benchmark dataset. We have demonstrated the effectiveness of these features over a large variety of supervised machine learning algorithms. Experiments suggest SVM is the best performing classifier on the selected dataset using the set of features. We believe the excellent performance of Hybrid LBP in terms of accuracy would motivate the researchers and practitioners to use it to identify protein structural class. To facilitate that, a classification model using Hybrid LBP is readily available for use at http://brl.uiu. ac.bd/PL/.

Protein-Ligand binding is accountable for managing the tasks of biological receptors that help to cure diseases and many more. So, binding prediction between protein and ligand is important for understanding a protein's activity or to accelerate docking computations in virtual screening-based drug design. ProteinLigand Binding Prediction requires three-dimensional tertiary structure of the target protein to be searched for ligand binding. In this paper, we've proposed a supervised learning algorithm for predicting ProteinLigand Binding which is a Similarity-Based Clustering approach using the same set of features. Our algorithm works better than the most popular and widely used machine learning algorithms.

\section{INTRODUCTION}

Protein tertiary structure comparison is very important in many applications of modern structural biology, drug design, drug discovery, in studies of protein-ligand binding, protein-protein interactions, and other fields. This is especially significant because the structure of a protein is more protected than the protein sequence (Chothia and Lesk, 1986). Many works have been done to find protein binding (Brady and Stouten, 2000). Comparison of protein structure has been done in many works of literature by alignment

*First two authors contributed equally 
of distance matrices (Holm and Sander, 1993), using iterated double dynamic programming (TAYLOR, 1999), using elastic shape analysis (Srivastava et al., 2016) and many other techniques. The most common way of comparing protein tertiary structure is to treat the protein as a three-dimensional object and superimpose one on another. Different distances are used to calculate the differences between the proteins.

The distance matrix of $\alpha$ carbon can be seen extensively used in (Holm and Sander, 1997; Singh and Brutlag, 1997) as a feature which represents the tertiary structure of a protein chain. This feature is used as a feature vector which represents the structure of a protein to measure either similarity or dissimilarity to measure and compare the feature vectors with one another in pattern recognition literature. A mapped two-dimensional feature matrix is created from the 3D coordinate data of protein. The intra-molecular distance is used to make the $\alpha$ carbon distance matrix which mirrors the tertiary structure of a protein and the conserved elements of the secondary structure in it. With an input matrix size of $\mathrm{N} \mathrm{x} \mathrm{N}$, the distance matrix based exact algorithms run in $O(N)$ time (Karim et al., 2015).

An image is basically a matrix of $\mathrm{N} x \mathrm{~N}$ dimension with corresponding data in each cell. Thus the distance matrix can be used as an image. Basically, three types of features can be generated from an image: pixel based, filter based and computationally generated features. Pixel-based features e.g histograms are simplistic and dependent on the capability of classification algorithms. Filter based methodologies transform the original image to use feature extraction methods. Refined algorithms are used to segment and other various algorithms are used to detect different features. Using ideas from computer vision and utilizing it in protein structure retrieval is not uncommon in the field. ProteinDBS server (Shyu et al., 2004) implement a similar approach in (Chi et al., 2005) by Chi et al. Texture features from the original size images and diagonally partitioned images were extracted by Chi et al. CoMOGrad and PHOG (Karim et al., 2015) also used images to extract their two novel feature whereas we are extracting histograms of local binary pattern images from the original image.

The human body uses protein for repairing tissues, making enzymes, hormones, and other biological chemicals. It is an essential building block of bones, muscles, cartilage, skin, and blood. On the other hand, a ligand is a material that has the potentiality to bind to and forms a composite with a biomolecule in order to carry out a biological function. In Protein-Ligand Binding, the ligand is usually a molecule which produces a signal by binding to a locus on a target protein. The binding typically results in a change of conformational isomerism (conformation) of the target protein. The evolution of the protein's responsibility depends on the development of specific sites which are designed to bind ligand molecules. Ligand binding ability is important for the management of biological functions. Ligand binding interactions change the protein state and function. Protein-Ligand Binding prediction is very important in many applications of modern structural biology, drug design, drug discovery, and other fields.

Many experimental techniques can be used to investigate various aspects of protein-ligand binding. X-ray crystallography, nuclear magnetic resonance(NMR), Laue X-ray diffraction, small-angle X-ray scattering and cryo-electron microscopy provide atomic-resolution or near-atomic-resolution structures of the unbound proteins and the protein-ligand complexes, which can be used to study the changes in structure and/or dynamics between the free and bound forms as well as relevant binding events. Although experimental techniques can investigate thermodynamic profiles for a ligand-protein complex, the experimental procedures for determination of binding affinity are laborious, time-consuming, and expensive. Modern rational drug design usually involves the HTS of a large compound library comprising hundreds or thousands of compounds to find the lead molecules, but this is still not realistic to use experimental methods alone. Different methods like Isothermal Titration Calorimetry (ITC) (Chaires, 2008), Surface Plasmon Resonance (SPR) (Patching, 2014), Fluorescence Polarization(FP) (Rossi and Taylor, 2011), Protein-Ligand Docking (Sousa et al., 2013), Free Energy Calculations (Steinbrecher and Labahn, 2010), etc are being used to predict ligand-binding prediction.

In this paper, we propose the combination of local binary pattern histogram, Gabor Filtered Local Binary Pattern Histogram, Separate Row Multiplication Matrix with Uniform Local Binary Pattern Histogram, neighbor Block Subtraction Matrix with Uniform Local Binary Pattern Histogram and Atom Bond features to be used for protein similarity measurement. We extract the distance matrix of $\alpha$ carbon of a protein from PDB file and use the distance matrix as an image to extract our first four features and Atom Bond is extracted from the PDB files. We have used a large variety of classification algorithms to test the extracted features. We are also going to show the results and comparative study of different implementation methodology of CoMOGrad and PHOG. The method we have proposed can produce a better result on some classification algorithm over the previous methods on the same benchmark. In 


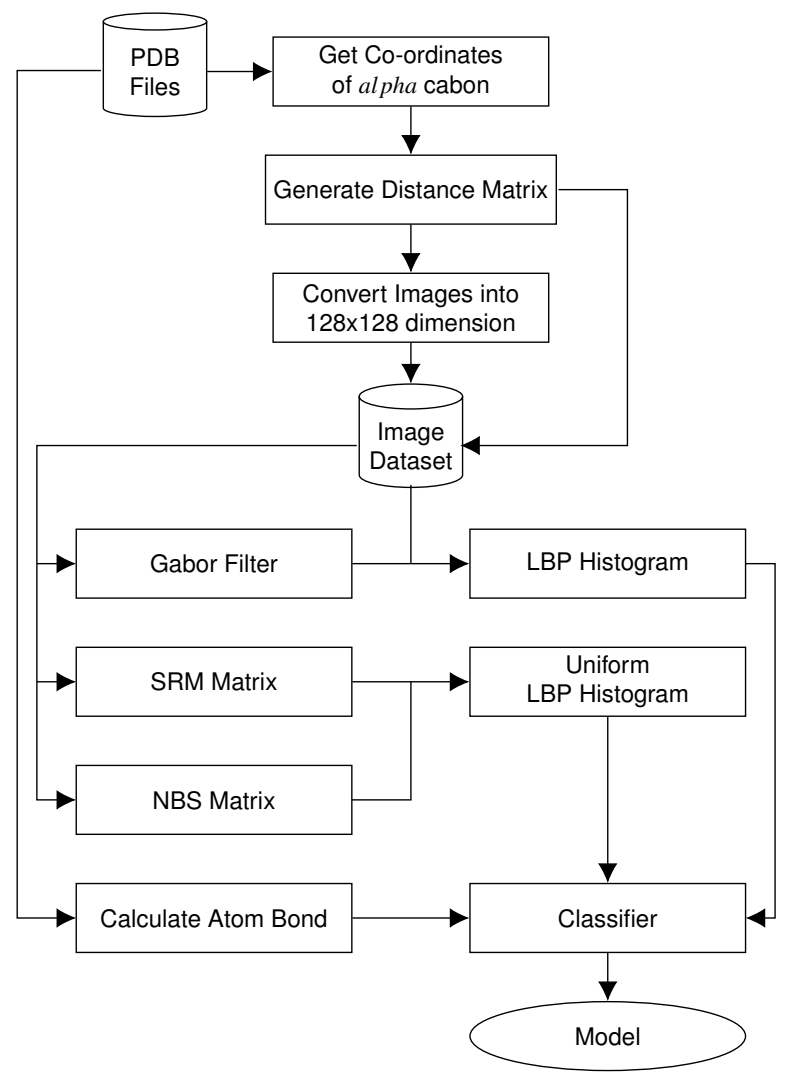

Figure 1. Block diagram of the methodology used in structural class prediction.

addition to that, we've proposed a supervised learning algorithm for predicting Protein-Ligand Binding which is a Similarity-Based Clustering approach using the same set of features. Our algorithm works better than the most popular and widely used machine learning algorithms. Our proposed method uses the features proposed in this paper.

\section{MATERIALS AND METHODS}

Our methodology is divided into two parts. Firstly, we have generated image based features using protein tertiary structures and performed feature analysis based on the prediction power on the structural class prediction problem. In this section, we present the materials and methods for both of the problems. For each of the problems the dataset, features, necessary algorithms and performance measurement is described accordingly.

\section{Structural Class Prediction}

In this section, we present the methodology of structural class prediction. Atom bond features are generated from the protein tertiary structures given as PDB files. Images are created from the distance matrix calculated using $\alpha$ carbon atom coordinates of the amino acids of the protein structures in the given dataset. From each image of protein, we have derived five features. Stratified Remove Folds was used to test the capability and efficiency of the dataset. There are in total seven different classes of protein structures. Synthetic minority over-sampling technique (SMOTE) is used to handle class imbalance problem. The block diagram of the methodology is given in Figure 1.

\section{Structural Class Prediction Dataset}

We have used 40 percent ID filtered subset of PDB-style files for SCOPe domains version 2.03 (Fox et al., 2013) as our dataset. It contains a total of 12119 PDB files. Each PDB files contains SCOP(e) concise classification string (sces) which respectively describes class, fold, superfamily, and family. In this paper, we are going to experiment only with the class of the protein. In the dataset, there are total seven protein 


\begin{tabular}{|c|c|}
\hline Class Name & Total Instances \\
\hline Small Proteins & 640 \\
\hline All $\alpha$ Proteins & 2195 \\
\hline$\alpha$ and $\beta$ proteins $(\mathrm{a} / \mathrm{b})$ & 3305 \\
\hline$\alpha$ and $\beta$ proteins $(\mathrm{a}+\mathrm{b})$ & 3006 \\
\hline Membrane and cell surface proteins and peptides & 204 \\
\hline All $\beta$ proteins & 1485 \\
\hline Multi-domain proteins $(\alpha$ and $\beta)$ & 219 \\
\hline
\end{tabular}

Table 1. Protein Classes and its Corresponding Instances

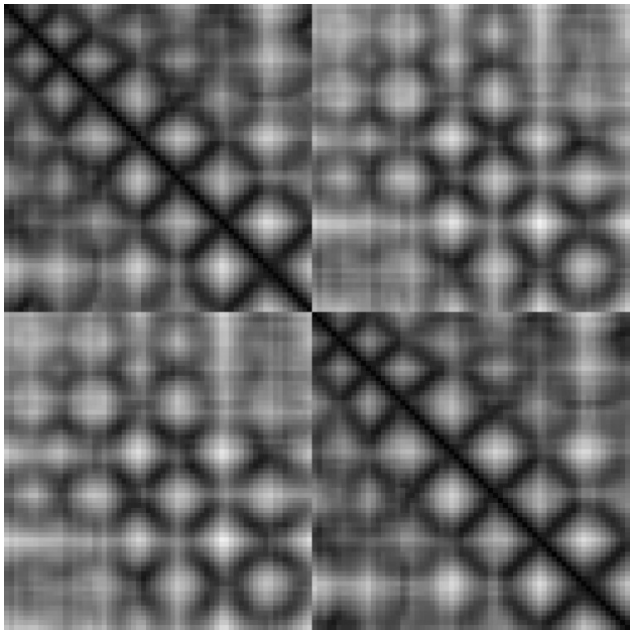

(a)

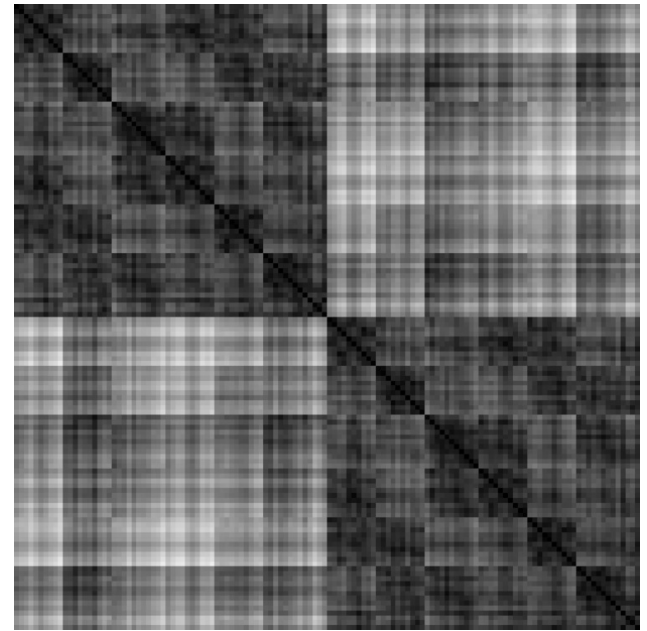

(b)

Figure 2. Sample images of protein structures after rescaling (a) showing diagonal and (b) symmetric textures.

structural classes. For benchmark analysis with CoMOGrad and Phog, the common PDB files were used as dataset. The common PDB files are total of 11052. The details of the protein structural classes are given in Table 1. This dataset is widely used as a benchmark in the literature for protein structural similarity prediction (Karim et al., 2015).

\section{Image Generation}

We have generated images of protein structures according to the methodology described in CoMOGrad and PHOG (Karim et al., 2015). Only $\alpha$ carbons of the amino acids in the protein structure are considered for image generation. From the three dimensional coordinates of the $\alpha$ carbon atoms, a distance matrix is generated by taking the Euclidean distance among all pairs. This distance matrix converts a 3D structure of a protein to a 2D matrix. Euclidean distance is applied as the distance measure because it exceeds the popularly applied costly alignment distance measure of $\alpha$ carbon distance matrices. Thus only half of the matrix contains redundant information due to symmetry. The matrix can be further regarded as an image.

\section{Scaling of Images}

The dimension of protein images is based on the total number of $\alpha$ carbon they have. So, every individual protein images are different from the other in dimension. Therefore, the images were scaled to the same dimension. CoMOGrad and PHOG have used Bi-cubic interpolation and wavelet transform to scale all the protein images into $128 \times 128$ dimension (Karim et al., 2015). During the Bi-cubic interpolation step, most of the images were in $128 \times 128$ dimension so in the wavelet transform step they scaled all the images to that dimension. Thus, we have directly scaled the images to $128 \times 128$ dimension. We have used both real and scaled images to examine the differences in their predictive power. Sample rescaled images of protein structures are given in Figure 2. 


\begin{tabular}{|l|l|l}
\hline 6 & 5 & 2 \\
\hline 9 & 4 & 2 \\
\hline 1 & 7 & 8 \\
\hline
\end{tabular}$\quad$ Threshold \begin{tabular}{|l|l|l|l|}
1 & 1 & 0 \\
\hline 1 & & 0 \\
$\begin{array}{ll}\text { Binary : 11001101 } \\
\text { (ClockWise) }\end{array}$ \\
0 & 1 & 1 \\
Decimal : 205
\end{tabular}

Figure 3. An example of basic LBP

\section{Feature Extraction}

We have generated five different feature groups. Our first four feature groups are different types of histograms and the fifth feature group is about the prognosis of the atoms. The histograms were taken from both scaled and unscaled images.

Local Binary Pattern Histogram Local binary pattern (LBP) histogram was first proposed by Ojala et al. (1994) and popularized by the work of Ojala et al. (2002). Local binary pattern computes the local representation of the texture of an image as a texture descriptor. Comparing each pixel with its neighboring pixels the local representation is created. The image is transformed into a grayscale image. In a $3 \times 3$ neighborhood, the center pixel value is calculated by comparing with its eight neighboring pixels. Each comparison gives a result of either 0 if the center pixel value is greater then the comparing neighbor pixel or 1 for the latter. A clockwise direction starting from the top-left one provides a binary number. The binary number is converted to a decimal number and the value is placed in the center pixel. LBP codes or Local Binary Patterns are the obtained binary numbers. An example of a basic Local Binary Pattern is given in Figure 3. After calculating the value for each pixel of the image, a histogram is calculated. A $3 x$ 3 neighborhood has $2^{8}=256$ possible patterns, thus the values range from 0 to maximum 255 in each pixel of the image. The total number of bins of the histogram is thus 256 . We would get 256 attributes from each image. We have used zero-padding technique to generate local binary pattern.

Gabor Filtered Local Binary Pattern Histogram (GfLBP-Hist) Gabor Filter is titled after Dennis Gabor. It is used for texture segmentation (Jain and Farrokhnia, 1991), optical character recognition (Jain and Bhattacharjee, 1992), edge detection (Mehrotra et al., 1992) etc. It is a linear filter which examines if there is any particular frequency content in the image in specific areas in a localized region throughout the point. In spatial and frequency domain, the Gabor filter has been determined to own optimal localization properties (Jain et al., 1997). Gabor filter lets a particular band of frequencies while discarding others and that is why it is designated as bandpass filter.It resembles the features of simple visual cortical cells (Dunn and Higgins, 1995). It provides the largest response where texture changes at edges and at points. The multiplication of a sinusoid and a Gaussian is called the Gabor filter.The filter has a real and an imaginary segment rendering orthogonal directions. The two segments can be set into a complex number or used individually.Various shapes, sizes and smoothness levels in an image can be detected by Gabor filter.

$$
g(x, y ; \lambda, \theta, \phi, \gamma)=\exp \left(-\frac{x^{\prime 2}+\gamma^{2} y^{\prime 2}}{2 \sigma^{2}}\right) \exp \left(\imath\left(2 \pi \frac{x^{\prime}}{\lambda}+\phi\right)\right)
$$

$$
g(x, y ; \lambda, \theta, \phi, \gamma)=\exp \left(-\frac{x^{\prime 2}+\gamma^{2} y^{\prime 2}}{2 \sigma^{2}}\right) \cos \left(2 \pi \frac{x^{\prime}}{\lambda}+\phi\right)
$$

$$
g(x, y ; \lambda, \theta, \phi, \gamma)=\exp \left(-\frac{x^{\prime 2}+\gamma^{2} y^{\prime 2}}{2 \sigma^{2}}\right) \sin \left(2 \pi \frac{x^{\prime}}{\lambda}+\phi\right)
$$

(Eq.1) is the complex version of the Gabor function and (Eq.2) and (Eq.3) are real and imaginary version respectively. The shape and size of the Gabor function is regulated by its five parameters. Here, $\lambda$ controls the wavelength of this sinusoid, $\theta$ is the angle of the normal to the sinusoid, $\phi$ is the phase shift of the sinusoid, $\gamma$ controls the aspect ratio, The spatial envelope or the standard deviation of the Gaussian is $\sigma$. For our experiments, we have used $\lambda=10, \theta=0, \phi=0, \gamma=0.02$ and $\sigma=5$. After applying the Gabor filter, LBP techniques are applied to the image to get 256 attributes. 


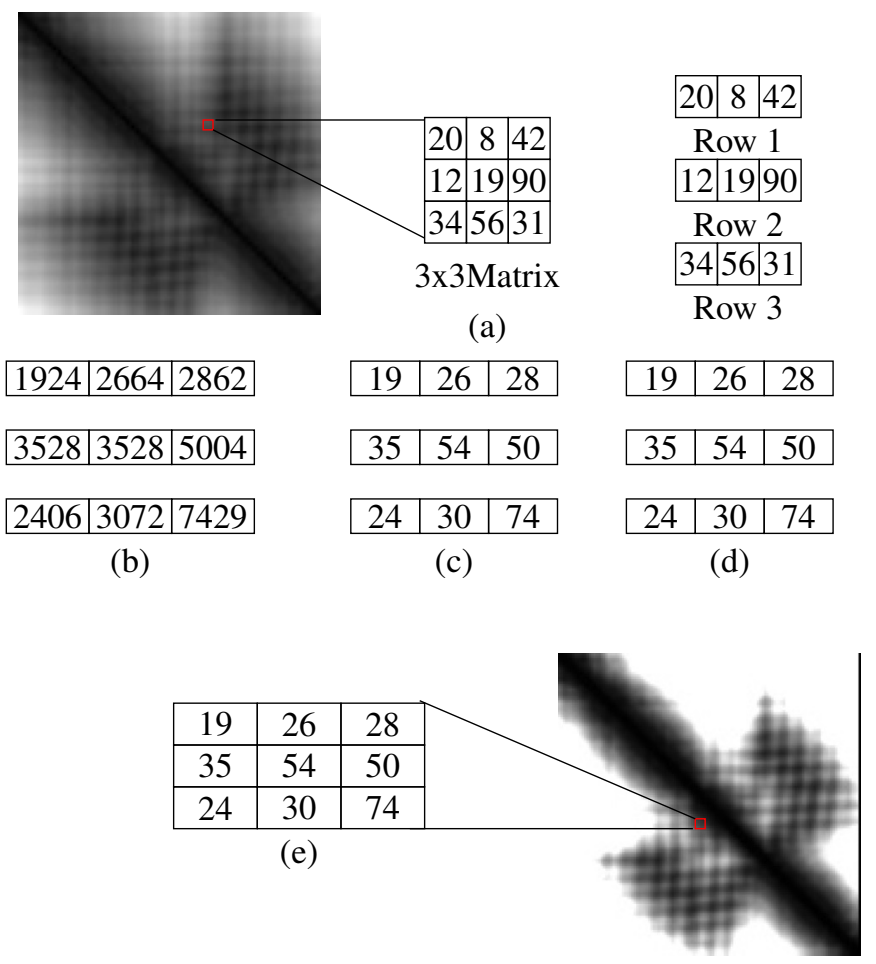

Figure 4. An example of Separate Row Multiplication Matrix with Uniform Local Binary Pattern Histogram (a) sample figure and matrix; (b) after multiplication; (c) \& (d) separation of the matrices and (e) finally showing the filtered image.

Atomic Bond Features First of all, we've identified unique atoms amidst all the protein PDB files. From each protein PDB file, we've counted occurrences of each atom. Then we've taken the percentage as features of each atom among all the atoms that each protein has. Then we've taken first 100 sequential atoms and used their atomic mass as the feature. Then we've counted the bond that each pair of atoms has in a particular protein using atomic distance based on a threshold value. Finally, we've taken the percentage as the feature of the bond of each unique pair of atoms among all the bonds that the protein has.

Separate Row Multiplication Matrix with Uniform LBP Histogram(SRMMat-ULBP-Hist) The image is split into $3 \times 3$ matrices. From each matrix, we get 3 rows with the dimension of $1 \times 3$. By multiplying each row with the same $3 \times 3$ matrix, we get three result matrix consisting of $1 \times 3$ dimension. Each cell is divided by 100 . The results are then put in the $3 \times 3$ matrix in accordance with the row numbers. The color intensity of an image is between 0 to 255 . So, if the value of any cell of the result matrix is greater than 255 , then the value is replaced with 255 . After applying this technique, the uniform local binary pattern is applied. From Figure 4, (a) presents a $3 \times 3$ section of matrix and the rows, (b) exhibits the result of multiplication, (c) shows the value after dividing by 100 , (d) shows the replacement result of value greater than 255 and (e) shows a $3 \times 3$ matrix section after SRM-Matrix transformation.

Another variation of the LBP is called uniform pattern (Ojala et al., 2002). Some binary patterns occur more generally in texture images. If the binary pattern comprises at most two $0-1$ or 1-0 transitions when the bit pattern is held circular then the pattern is called uniform. For instance, 01000000 has 2 transitions, 00000111 has 2 transitions which are uniform pattern on the other hand 01010100 has 6 transitions, 11001001 has 4 transitions which are not uniform. A neighborhood with the dimension of $3 \times 3$ has $2^{8}=256$ possible patterns with 58 of them being uniform. For estimating the histogram, every uniform pattern gets a separate bin while a single bin is allotted for all non-uniform patterns. Therefore, from a uniform binary pattern, we get the histogram of the total bin size of 59 . 


\begin{tabular}{|c|c|c|}
\hline Identifier & Feature Group Name & Number of Features \\
\hline A & LBP-Hist & 256 \\
\hline B & GfLBP-Hist & 256 \\
\hline C & Atom Bond & 116 \\
\hline D & SRMMat-ULBP-Hist & 59 \\
\hline E & NBSMat-ULBP-Hist & 59 \\
\hline
\end{tabular}

Table 2. Feature Groups

Neighbor Block Subtraction Matrix with Uniform LBP Histogram (NBSMat-ULBP-Hist) Blocks are of the same dimension, $3 \times 3$. Two blocks of matrices are considered neighbors for this method if the center cells are neighboring. Because of this, the value of the last two columns of the first block and the first two columns of the second block are the same. The two blocks of matrices are subtracted and the result is set in the place of the first block. If any of the cells have any negative number, then 0 is placed instead of the negative value. The replacing of value is made because the histogram bin begins from zero. Uniform local binary pattern is then used to compute the histogram.

Summary of all the feature groups used in this paper is given in Table 2.

\section{Handling Imbalance in Data}

From Table 1 it can be noted that the classes are imbalanced. To balance the classes, we have used Synthetic Minority Over-sampling Technique (SMOTE) (Chawla et al., 2002). The percentage of SMOTE indicates that how many more instances would be generated. We have over-sampled our instances close to the highest number of instances among all the classes. If $x$ denotes the highest number of instances among all the classes and $y$ denoted by a class which we will SMOTE then the expression for the percentage calculation is $\frac{x-y}{y} * 100$. We have used 5 nearest neighbors to generate the over-sampled instances.

It is to be noted that SMOTE was used on only train dataset after separating the train and test dataset. So, there are no artificial instances in test dataset.

\section{Classifiers Used}

We have used five classifiers for the analysis of features applied to solve structural class prediction problem: K-Nearest Neighbor (KNN), Naive Bayesian Classifier, Support Vector Machines (SVM), Adaptive Boosting (AdaBoost) and Random Forest. A concise description of the classifiers is given in this section.

K-Nearest Neighbor (KNN) K-nearest neighbor algorithm (KNN) (Aha et al., 1991) is a similaritybased classification technique. It is a lazy classification technique. Distance metrics are used for each instance of the whole dataset for calculating the $K$ nearest neighbors. The labels of the nearest neighbors decide the label of the test instances. It works poorly for high dimensional data. Euclidean distance, Hamming distance, Manhattan distance, Minkowski distance, Tanimoto distance and Jaccard distance are used for similarity measures.

Naive Bayesian Classifier Naive Bayesian classifier (Maron, 1961) is based on probabilistic inference of samples observed where the decision variable and the features form a very naive structure of Bayesian Network. Naive Bayesian classifiers work best for image recognition and text mining.

Support Vector Machine (SVM) Support Vector Machine (Cortes and Vapnik, 1995) works by creating and separating hyperplane for a given dataset by sampling different classes which are separated by maximum width.

Adaptive Boosting (AdaBoost) Adaptive Boosting classifier (Freund and Schapire, 1997) is a metaclassifier which aims to make a strong classifier using a set of weak classifiers. The classifiers whose performance is marginally better than random classifiers are called weak classifiers.

Random Forest Random Forest (Ho, 1995) is an ensemble classifier. A decision tree is created in each iteration with features taken randomly. It samples selected features using bootstrap aggregating. 


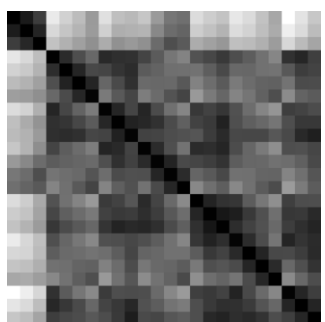

(a)

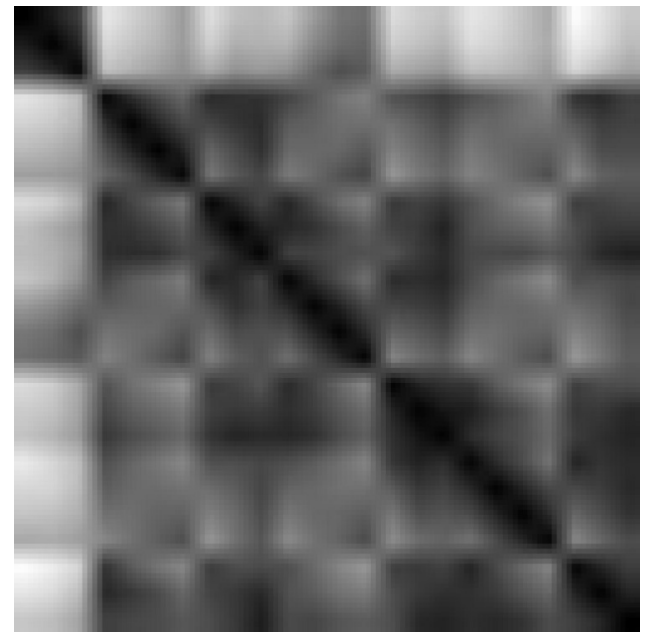

(b)

Figure 5. Images of ligand structures: (a) non-scaled and (b)scaled.

\section{Ligand-binding Prediction}

Protein-Ligand Binding prediction is a binary class classification problem. We've used Image-Based Features for each Protein and Ligand dataset. Our methodology learns threshold values from the training data and uses these in test data prediction. We have used the same set of features that were generated and analyzed for the structural class prediction problem to solve the ligand-binding problem. In this section, we present the necessary materials and methods that were used for the ligand-binding problem.

\section{Ligand-Binding Dataset}

We've used Computer Vision and Pattern Discovery for Bioimages Group @ BII as our dataset. In our dataset, 3000 protein-ligand complexes were determined experimentally with 3D structures available. Each protein and its ligand are of one-to-one correspondence, i.e. they can bind to each other and make Protein-Ligand complex. The dataset has 3000 pairs of protein and ligand where the same name/ID of protein and ligand interacts/binds with each other.

We've used OpenCV (Bradski and Kaehler, 2008) library to create images from PDB files. For protein, we've considered the coordinates of only the alpha-carbons to generate the distance matrix to create image. Because alpha-carbon can represent the structural information of protein quite well. But the given ligands were small in terms of atom number. So, while creating ligand images, we've considered all the atom's co-ordinates for generating distance matrix.

Among the PDB files, 33 ligands have only one atom, which will create a $1 \times 1$ image having no significance for feature extraction. So, we had to compromise those 33 ligands as well as 33 corresponding proteins from training. Figure 5 shows a sample non-scaled and scaled image of a ligand.

\section{Handling Imbalance}

The given dataset has only positive instances (the pairs of protein and ligand where they bind with each other). But there were no negative instances (the pairs of protein and ligand where they do not bind with each other). The missing negative instances have created our dataset highly imbalanced. To overcome this imbalance, we've generated negative instances in two different ways.

1. Random Negative Undersampling: We have 2967 protein PDB and 2967 ligand PDB where 8803089 pairs are possible. Among these, 2967 pairs are given as positive instances and the rest 8800122 pairs are unknown/unseen instances. From the unseen pairs, we've taken 2967 pairs randomly as negative instances to make our dataset balanced.

2. Clustering-Based Undersampling: Using the positive instances (2967 pairs), we've created 10 clusters. Then we've searched for 2967 unseen pairs randomly as negative instances where they belong to those 10 clusters. We've made sure that each cluster has exactly the same number of positive and negative instances to make the dataset balanced (See Figure 6). 


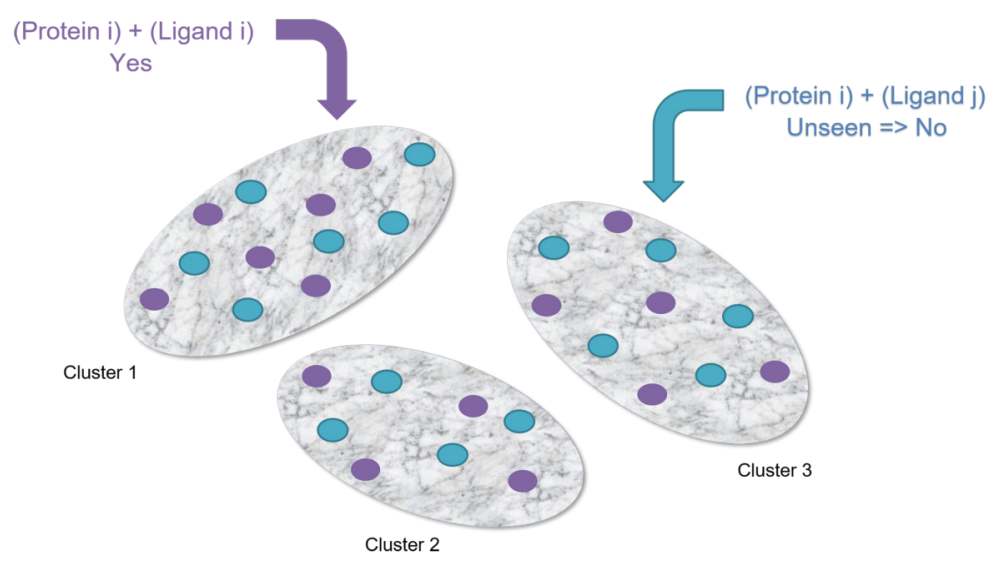

Figure 6. Clustering-Based Undersampling.

\section{Similarity Based Classifier}

We've developed a similarity-based clustering method to predict the binding class. Distance is used to measure similarity. Our methodology is given in Figure 7 and the pseudo-code in Algorithm 1.

Data: A pair $(p, l)$, a protein structure and ligand structure in PDB format

Result: Decision, whether they will interact or not

1 for all proteins and ligands do

2 generate images \& extract features

3 end

4 for each of the given pairs of protein-ligand do

$\mathbb{N P} \leftarrow k$-NEARESTPROTEINS $(p)$ of the given protein

$\mathbb{R L} \leftarrow k$-RELATEDLIGANDS $(\mathbb{N P})$

$d_{l} \leftarrow$ distance between given ligand, $l \& \mathbb{R} \mathbb{L}$

if $d_{l}<$ threshold $d_{l}$ then

$v_{l} \leftarrow$ vote for positive bind

else

$v_{l} \leftarrow$ vote for negative bind

end

$\mathbb{N L} \leftarrow k$-NEARESTLIGANDS $(l)$ of the given ligand

$\mathbb{R P} \leftarrow k$-RELATEDPROTEINS $(\mathbb{N L})$

$d_{p} \leftarrow$ distance between given protein, $p \& \mathbb{R} \mathbb{P}$

if $d_{p}<$ threshold $d_{p}$ then

$v_{p} \leftarrow$ vote for positive bind

else

$v_{p} \leftarrow$ vote for negative bind

end

$v \leftarrow$ majority voting between $\left(v_{l}, v_{p}\right)$

22 end

23 return $v$

Algorithm 1: Similarity-based clustering algorithm.

From the PDB dataset of proteins and ligands, firstly we have generated images and converted to $128 \times 128$ images for each protein and ligand. From these images, we have generated 2 different features.

1. CoMOGrad and PHOG: CoMOGrad stands for Co-occurrence Matrix of the Oriented Gradient of Distance Matrices and PHOG stands for Pyramid Histogram of Oriented Gradient (Karim et al., 2015). This methodology also uses the $\alpha$ carbon distance matrix of protein. The dimension of all distance matrix is converted to $128 \times 128$. In CoMOGrad, the gradient angle and magnitude is computed from the distance matrix and the values are quantized. Quantization is a compressing technique which compresses a range of values to a single quantum value. In this methodology, the 


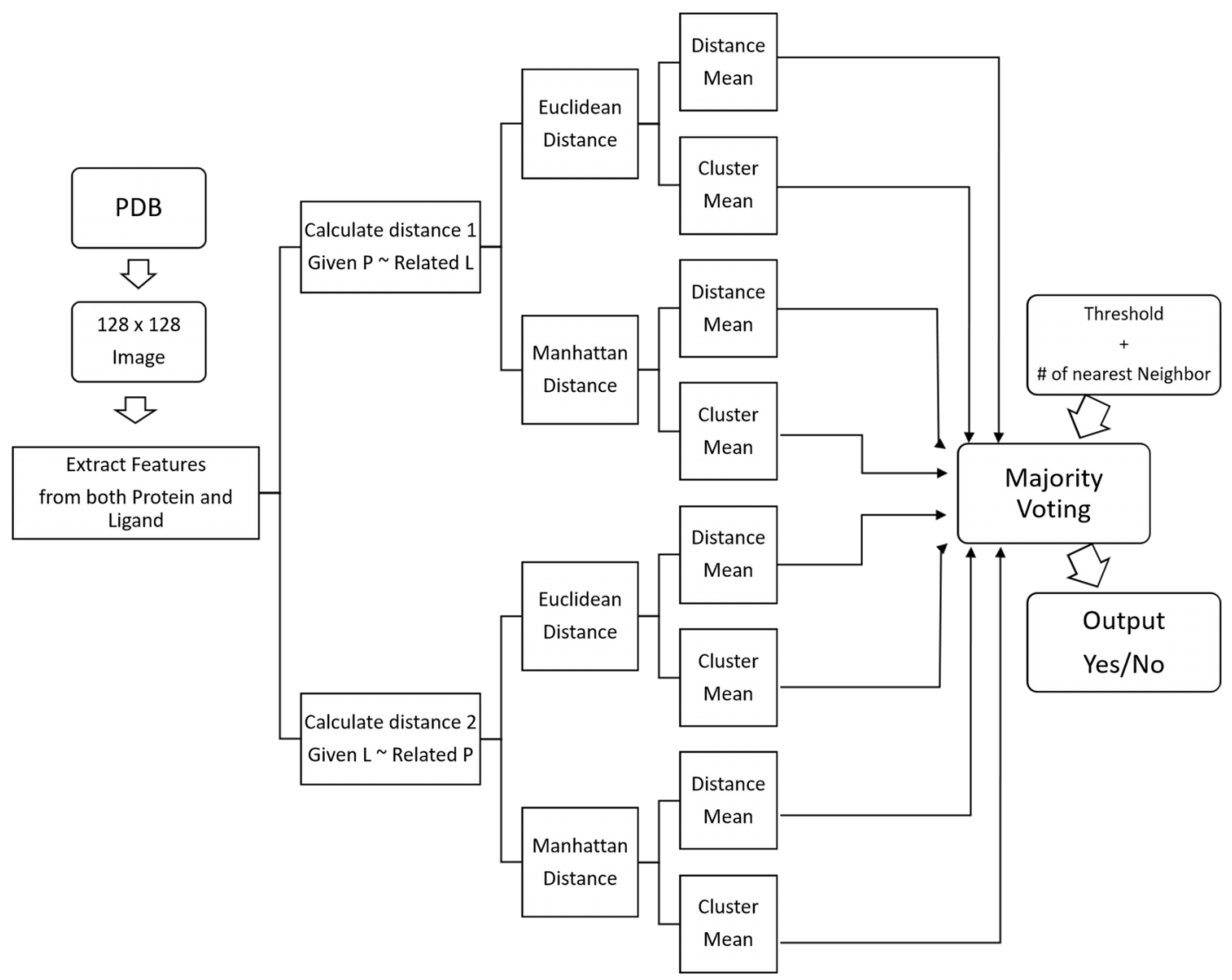

Figure 7. Block Diagram of Similarity-Based Clustering. 


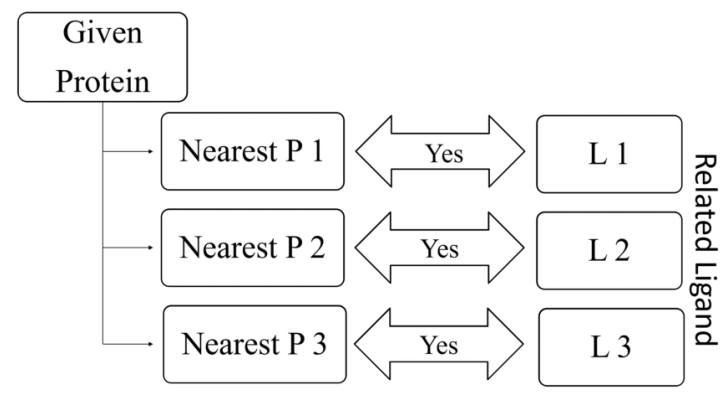

Figure 8. Relation between given protein and related ligands.

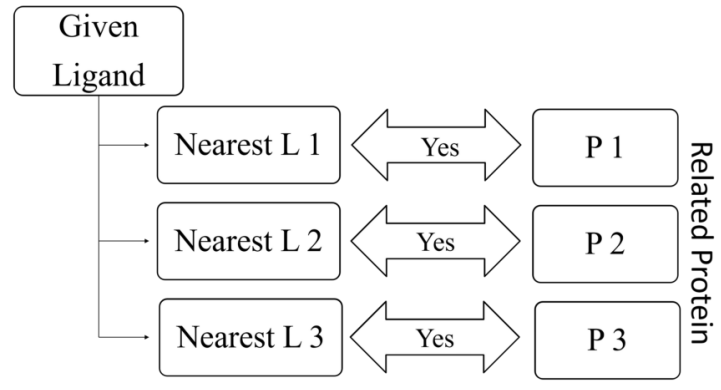

Figure 9. Relation between given ligand and related proteins.

values are quantized to 16 bins which produce a co-occurrence matrix which is $16 \times 16$ matrix The matrix is converted into a vector of size 256. Quadtree from the distance matrix is created with the desired level in PHOG. Gradient Oriented Histogram of each node is calculated with the preferred number of bins and bin size. In gradient oriented histogram an image is divided into small sub-images called cells and histogram of edge orientations are accumulated within the cell. The combined histogram entries are used as the feature vector describing the object. Total features which are the multiplication of total nodes and number of bins are incorporated in the vector with the size of the total number of features. The vector is normalized by dividing it with the sum of its components.

2. Hybrid Local Binary Pattern (Hybrid LBP): Local Binary Pattern (Ojala et al., 1994) is a procedure of local binary pattern histogram. We have used all the five feature groups described in the last section for structural class prediction problem.

Distance can only be calculated between proteins or between ligands. We've used K-nearest neighbor and Clustering method to calculate these distances.

1. RelatedLigands $(\mathbb{N P})$ : For a given Protein, find K-nearest proteins. The ligands those binds with the above nearest proteins are the Related Ligands for the given protein (See Figure 8).

2. RELATEDPRoteins(NL): For a given Ligand, find K-nearest ligands. The proteins those binds with the above nearest ligands are the Related Proteins for the given ligand (See Figure 9).

To find the distances between pairs of ligands and proteins are calculated using Euclidean and Manhattan distances. Threshold is the boundary between similarity and dissimilarity in terms of distance. If distance is less than the threshold, then prediction is positive similarity, else the prediction is negative similarity. The threshold of each category of distances is the average distance based on the number of nearest neighbors. Measuring distance has been done in two ways. One method is to get the cluster mean of k-RelatedLigands/RelatedProteins, then measure the distance from the GivenProtein/GivenLigand. Another method is to measure distances between the GivenProtein/GivenLigand and k-RelatedLigands/RelatedProteins, then take the average of those distances as the final distance.

For a given pair of Protein and Ligand, we want to predict if the will bind with each other or not. For measuring distance $d_{l}$, from the given protein, we searched for k-nearest proteins and found the $\mathrm{k}$ related 
ligands accordingly. Then we've calculated the distance using the above mentioned methods. Then we've taken the vote for the binding class by all categories of distances based their thresholds. Then finally, we've used majority voting mechanism to predict the binding class.

\section{Hyperparameters}

There are some hyperparameters of our proposed method.

1. Number of nearest neighbors: Our algorithm's prediction accuracy is highly dependent on the number of nearest neighbors for finding both RELATEDLIGANDS $(\mathbb{N P})$ and RELATEDPROTEINS $(\mathbb{N L})$. We've used 5 and 3 nearest neighbors in this experiment.

2. Threshold: This is the threshold of distance for determining whether two proteins or two ligands are similar or not. For a higher value of threshold, there is a higher possibility for our algorithm to predict positive binding class for the majority of the Protein-Ligand pairs. And the lower the threshold is, the higher is the possibility of negative binding class prediction. We've taken the average of distances among 3 nearest neighbors as our threshold for each category of the distances. In terms of setting the threshold values, distances of each training sample from the whole training data was measured. While doing so, negative pairs of protein-ligand were considered when positive pair of protein-ligand was given for getting distances. Similarly, positive pairs of protein-ligand were considered when negative pair of protein-ligand was given. Average of these distances was taken as threshold values.

\section{RESULTS AND DISCUSSION}

This section is the description of our experiments performed in this study. Some of the experiments were carried out in a personal desktop computer having Intel Core i3 and 4 GB RAM and others were experimented in a Computing Machine provided by CITS, United International University which was equipped with 8 core processors each having a Dell R 730 Intel Xeon Processor (E5-2630 V3) with $2.4 \mathrm{GHz}$ speed and 18.5 GB memory. Java language was used for data preprocessing including feature generation using OpenCV software library, negative data generation and data merging using Eclipse IDE with Java 8 standard edition. Python language was used to implement our algorithm using the Spyder IDE. Weka tool was used to run the traditional classification algorithms for the comparison with our algorithm. 10 -fold cross-validation method has been used to get the performance of our model.

We also used Scale-invariant feature transform(SIFT)(Lowe, 2004) methodologies in our experiments. Each descriptor has a 128-dimensional feature vector. The number of the descriptors of SIFT from every image is not specific so we cannot use traditional machine learning techniques. Hence to apply traditional machine learning procedure and specify the feature vector, we split the image into 16 slices and took one descriptor from each of the slice images. Therefore we got 2048 number of attributes $(8 \times 16)$ from each image. We tested the dataset with the same classifiers mentioned in this paper. The results didn't turn up to be better or close to our proposed methodology in this literature. The result of accuracy on different classifiers using SIFT is given in supplementary file 01.

We've tried outlier detection method to handle the absence of negative data in Ligand-Binding dataset. But it gave overfitting problem. Details are in Supplementary file 05.

\section{Analysis of Features}

A different sets of parameters were used for each classifier used in this research. A linear searching was used with no distance weighting for KNN. In the case of the Naive Bayesian Classifier, SVM, a polynomial kernel was used with $c=1.0$ and $\varepsilon=1.0 w^{-2}$. Data was normalized before supplying to the classifier. J48 decision tree classifier was used in Adaboost classifier as the weak base classifier. Classifier number of iterations was set to 100 for Random Forest.

We've used the "StratifiedRemoveFolds" filter which is available in Weka for creating Train and Test set. This is a supervised instance filtering process that takes a dataset and outputs a specified fold for cross-validation Kohavi (1995). We've taken one random stratified fold out of 10 stratified folds as a Test set. The Train set has been generated by applying SMOTE to the rest of the 9 folds for Class balancing. Then we've tested the performance of the features shown in Table 2 and different combinations of them using these Train \& Test set. Accuracy scores of the feature groups are given in Table 3. Other performance metrics results (sensitivity, specificity, f1 score) can be found on supplimentary file 7 . No 


\begin{tabular}{|l|l|l|l|l|l|l|}
\hline Image & Feature & \multicolumn{5}{|c|}{ Classifiers } \\
\cline { 3 - 7 } & Type & KNN & $\begin{array}{l}\text { Naive } \\
\text { Bayesian }\end{array}$ & SVM & $\begin{array}{l}\text { Adaboost } \\
\text { (J48) }\end{array}$ & $\begin{array}{l}\text { Random } \\
\text { Forest }\end{array}$ \\
\hline Scaled & A & 50.81 & 50.63 & 69.16 & 68.62 & 75.13 \\
\hline $\begin{array}{l}\text { Non } \\
\text { Scaled }\end{array}$ & A & 53.88 & 34.53 & 68.44 & 72.15 & 75.85 \\
\hline Scaled & $\mathrm{B}$ & 21.97 & 8.58 & 17.45 & 24.86 & 30.83 \\
\hline $\begin{array}{l}\text { Non } \\
\text { Scaled }\end{array}$ & $\mathrm{B}$ & 30.83 & 25.40 & 23.23 & 37.25 & 42.85 \\
\hline & $\mathrm{C}$ & 15.82 & 5.60 & 25.76 & 36.07 & 39.51 \\
\hline Scaled & $\mathrm{D}$ & $\mathbf{6 9 . 4 3}$ & 48.01 & 67.63 & 70.97 & 74.77 \\
\hline Scaled & $\mathrm{E}$ & 61.30 & $\mathbf{5 6 . 2 3}$ & 64.73 & 72.60 & 74.05 \\
\hline $\begin{array}{l}\text { Non } \\
\text { Scaled }\end{array}$ & AB & 51.71 & 32.45 & 68.89 & 71.33 & 73.50 \\
\hline $\begin{array}{l}\text { Non } \\
\text { Scaled }\end{array}$ & ABC & 27.30 & 32.27 & 68.62 & 69.80 & 74.23 \\
\hline $\begin{array}{l}\text { Non } \\
\text { Scaled + } \\
\text { Scaled }\end{array}$ & ABCD & 41.77 & 33.36 & 74.68 & 72.87 & 77.84 \\
\hline $\begin{array}{l}\text { Non } \\
\text { Scaled + } \\
\text { Scaled }\end{array}$ & ABCDE & 50.27 & 34.53 & $\mathbf{7 7 . 2 1}$ & $\mathbf{7 5 . 2 2}$ & $\mathbf{7 8 . 6 6}$ \\
\hline
\end{tabular}

Table 3. Classifier accuracies for different types of features and groups of features.

Cross-Fold Validation was performed here as the performance of one Test set is enough to find the best-performing group combination of features. The highest percentage of correctly classified instances achieved for each of the classifiers are indicated by the boldly faced values of the table.

After running the experiments for our five feature groups ABCDE classifies the highest percentage of correct instances in Random Forest, Adaboost(J48) and SVM among all other feature groups. Feature scaled E and D individually provides the highest accuracy in Naive Bayesian and KNN. As the whole combination of all feature groups, accuracy gives the highest percentage than any other feature group, thus we conclude that the best-performing feature group combination is ABCDE and the best classifier is Random Forest classifier.

\section{Effectiveness in structural class prediction}

In this section, we compare the performance of our proposed method with CoMOGrad and PHOG (Karim et al., 2015). For comparison with our methodology in this literature, we applied CoMOGrad and Phog techniques and Wavelet and Pyramid Histogram techniques in our dataset of 11052 instances. We conducted experiments with different classifiers using the same parameters as we did for feature analysis with the feature groups. We've discussed in the Analysis of Features section that we've tested our feature groups using StratifiedRemoveFolds and finally selected ABCDE as our best feature group and no CrossFold Validation was done. After selecting the feature group, we have had to apply Cross-Fold Validation to use individual observation exactly once for validation. For performing 10-Fold Cross-Validation, we've generated 10 different folds using "StratifiedRemoveFolds" and in 10 iterations, one fold has been kept as Test set and SMOTE has been applied to the other 9-folds to get the Train set of the running iteration. The result of performance metric(accuracy, sensitivity, specificity and f1 score) for each stratified iteration is given in supplimentary file 2 for HybridLBP and supplimentary file 3 for ComogPHOG. SMOTE hasn't been applied before splitting the data for cross-validation to avoid the presence of artificial data in the test set. Average scores of the 10 iterations are the actual score for comparing performance. This cross-validation was applied to all benchmark dataset along with ours to establish a valid comparison. The results are given in Table 4. From Table 4 it can be comprehended that our feature group ABCDE outperforms CoMOGrad and PHOG in Random Forest and Adaboost \& SVM. CoMOGrad and PHOG surpassed our feature groups in KNN, Naive Bayesian. It can be noted that the combination of our feature groups are three-fourths of CoMOGrad and PHOG. It also can be discerned that the accuracy percentage 


\begin{tabular}{|c|c|c|c|c|c|c|}
\hline \multirow{2}{*}{$\begin{array}{c}\text { Performance } \\
\text { Metric }\end{array}$} & Features & \multicolumn{5}{|c|}{ Classifiers } \\
\cline { 3 - 7 } & & KNN & $\begin{array}{c}\text { Naive } \\
\text { Baysian }\end{array}$ & SVM & $\begin{array}{l}\text { Adaboost } \\
(\text { J48) }\end{array}$ & $\begin{array}{c}\text { Random } \\
\text { Forest }\end{array}$ \\
\hline \multirow{2}{*}{ Accuracy } & Karim et al. (2015) & $\mathbf{6 8 . 0 3}$ & $\mathbf{5 6 . 4 0}$ & 76.52 & 72.50 & 74.27 \\
\cline { 2 - 7 } & This paper & 51.37 & 35.35 & $\mathbf{7 7 . 2 7}$ & $\mathbf{7 6 . 2 5}$ & $\mathbf{7 6 . 7 6}$ \\
\hline \multirow{2}{*}{ Sensitivity } & Karim & $\mathbf{6 8 . 0 4}$ & $\mathbf{5 6 . 4 1}$ & 76.53 & 72.5 & 74.28 \\
\cline { 2 - 7 } & This paper & 51.37 & 35.34 & $\mathbf{7 7 . 2 8}$ & $\mathbf{7 6 . 2 5}$ & $\mathbf{7 6 . 7 6}$ \\
\hline \multirow{2}{*}{ Specificity } & Karim et al. (2015) & $\mathbf{9 2 . 9 8}$ & $\mathbf{8 9 . 4 9}$ & 94.64 & 92.78 & $\mathbf{9 3 . 4 1}$ \\
\cline { 2 - 7 } & This paper & 92.93 & 84.09 & $\mathbf{9 5 . 0 4}$ & $\mathbf{9 3 . 0 2}$ & 93.34 \\
\hline \multirow{2}{*}{ F1 Score } & Karim et al. (2015) & $\mathbf{6 9 . 1 5}$ & $\mathbf{5 6 . 6 3}$ & 77.28 & 72.67 & 74.36 \\
\cline { 2 - 7 } & This paper & 54.21 & 31.88 & $\mathbf{7 8 . 2 8}$ & $\mathbf{7 6 . 2 3}$ & $\mathbf{7 6 . 7 7}$ \\
\hline
\end{tabular}

Table 4. Comparison of the proposed features in this paper with Karim et al. (2015) for structural class prediction.

in SVM is higher than all the classifier results. Thus, our novel features can classify more instances than CoMOGrad and PHOG.

We have revealed the precedence of our methodology over CoMOGrad and PHOG (Karim et al., 2015).

\section{Effectiveness in ligand-binding prediction}

Sensitivity is the true positive rate regarding the positive instances. As we had to generate the negative data artificially, sensitivity is the actual scale of performance measuring as the positive data were the actual data. Using the thresholds gained using the negative data, the sensitivity of our algorithm is very good comparing to other existing algorithms shown in Table 5 and Figure 10.

\begin{tabular}{|c|c|c|c|c|c|c|c|}
\hline $\begin{array}{l}\text { Performance } \\
\text { Metric }\end{array}$ & Features & $\begin{array}{c}\text { Adaboost } \\
\text { (J48) }\end{array}$ & $\begin{array}{l}\mathrm{KNN} \\
(5)\end{array}$ & $\begin{array}{c}\text { Random } \\
\text { Forest }\end{array}$ & SVM & $\begin{array}{c}\text { Naive } \\
\text { Baysian }\end{array}$ & $\begin{array}{c}\text { Our } \\
\text { Method (3) }\end{array}$ \\
\hline \multirow{3}{*}{ Sensitivity } & $\begin{array}{l}\text { HybridLBP } \\
\text { (random) }\end{array}$ & $41.90 \%$ & $36.30 \%$ & $21.90 \%$ & $33.70 \%$ & $43.10 \%$ & $66.21 \%$ \\
\hline & $\begin{array}{l}\text { HybridLBP } \\
\text { (cluster) }\end{array}$ & $52.90 \%$ & $48.70 \%$ & $53.40 \%$ & $51.00 \%$ & $37.60 \%$ & $65.82 \%$ \\
\hline & $\begin{array}{l}\text { ComogPHOG } \\
\text { (random) }\end{array}$ & $95.20 \%$ & $47.60 \%$ & $16.10 \%$ & $29.70 \%$ & $11.30 \%$ & $50.68 \%$ \\
\hline \multirow{3}{*}{ Specificity } & $\begin{array}{l}\text { HybridLBP } \\
\text { (random) }\end{array}$ & $41.20 \%$ & $47.20 \%$ & $18.20 \%$ & $39.00 \%$ & $49.60 \%$ & $42.19 \%$ \\
\hline & $\begin{array}{l}\text { HybridLBP } \\
\text { (cluster) }\end{array}$ & $55.80 \%$ & $58.00 \%$ & $62.20 \%$ & $56.90 \%$ & $65.50 \%$ & $42.19 \%$ \\
\hline & $\begin{array}{l}\text { ComogPHOG } \\
\text { (random) }\end{array}$ & $3.10 \%$ & $47.50 \%$ & $13.00 \%$ & $38.80 \%$ & $83.90 \%$ & $59.85 \%$ \\
\hline \multirow{3}{*}{ F1 Score } & $\begin{array}{l}\text { HybridLBP } \\
\text { (random) }\end{array}$ & $41.80 \%$ & $38.40 \%$ & $21.50 \%$ & $34.60 \%$ & $44.60 \%$ & $59.06 \%$ \\
\hline & $\begin{array}{l}\text { HybridLBP } \\
\text { (cluster) }\end{array}$ & $53.70 \%$ & $51.10 \%$ & $55.80 \%$ & $52.50 \%$ & $43.70 \%$ & $\mathbf{5 8 . 8 3 \%}$ \\
\hline & $\begin{array}{l}\text { ComogPHOG } \\
\text { (random) }\end{array}$ & $65.20 \%$ & $47.60 \%$ & $15.80 \%$ & $31.10 \%$ & $17.80 \%$ & $53.11 \%$ \\
\hline
\end{tabular}

Table 5. Sensitivity, Specificity and F1 score Comparison among different methods for ligand-binding prediction.

We have generated three different datasets based on three different features. Hybrid LBP gives 736 long feature vectors from protein images and 677 long feature vectors from ligand images. So, for one protein-ligand pair we've got 1413 (736+677) attributes and one Binding Class value as one instance. The above mentioned two types of negative data (random and Clustering-Based Undersampling) were generated using Hybrid LBP for balancing the data. CoMOGrad and PHOG gives 1021 or 1020 long 


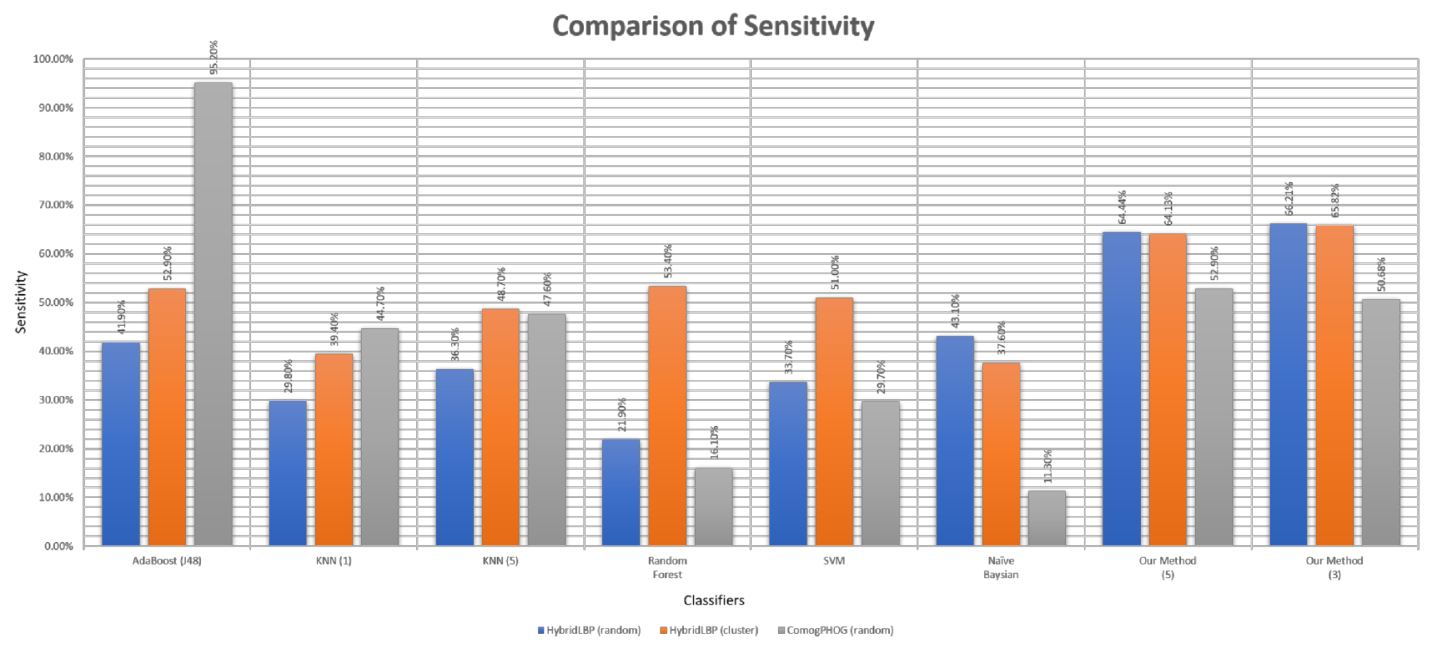

Figure 10. Barplot showing the performance of different algorithms on ligand-binding dataset.

feature vectors from protein image, but for ligand images, it gives 1020 long feature vectors. We assumed "0" as the last feature in protein where features were 1020 long, to make it a 1021 long feature. So, for one protein-ligand pair we've got $2041(1021+1020)$ attributes and one Binding Class value as one instance. Random negative undersampling was used in CoMOGrad and PHOG but Clustering-Based Undersampling was not possible as some clusters couldn't get any unseen pairs of protein and ligand. Our method was used based on 3 nearest neighbors and shown on the above table and chart.

Accuracy is not shown because it is dependent on the artificial negative data. However, specificity is shown to correlate with sensitivity, not to judge the algorithms as it is a true negative rate. On the other hand, the F1 score is also valuable because of being a harmonic mean of sensitivity \& precision where both scores are based on positive data. For more details, please check the supplementary file 06.

We can see that AdaBoost works better than our algorithm in terms of sensitivity in ComoGrad and PHOG dataset. Because Ligand data were so small in terms of number of atoms that ComoGrad and PHOG gave zeros for most of the ligands. This is an overfitting problem as the high sensitivity is an offset by low specificity. But our algorithm's overall performance is better than other machine learning algorithms in the three different feature datasets.

\section{CONCLUSIONS}

In this paper, we showed how accurately we can detect protein classes using the combination of different image-based feature groups generated from protein images. We also propose a simple similarity-based clustering method to predict Protein-Ligand Binding without using deep-learning or neural-networks. This simple distance-based algorithm is quite effective compared to complex machine learning algorithms. Our main limitation was the missing negative data. If we had the actual negative data, we could've determined the perfect thresholds for each category of distances, and that would give us more accurate prediction. Another problem was the dimensions of small Ligands as we're using image-based features. As the advancement of deep learning, neural network, and many other deep learning techniques are being used to classify images, many remarkably interesting applications can be made. For our future advancement, we wish to introduce new features to improve accuracy, use new tools and explore other fields of computer vision such as human emotion detection. Besides, we will try to extract some unique features from the Ligand dataset so that the dimensionality problem doesn't affect our Protein-Ligand binding prediction.

\section{ACKNOWLEDGMENTS}

We thank Rezaul Karim for sharing the SQL dataset files and algorithms to generate Distance Matrix from PDB files for CoMOGrad and PHOG. 


\section{REFERENCES}

Aha, D. W., Kibler, D., and Albert, M. K. (1991). Instance-based learning algorithms. Machine learning, 6(1):37-66.

Bradski, G. and Kaehler, A. (2008). Learning OpenCV: Computer vision with the OpenCV library. "' O’Reilly Media, Inc.”.

Brady, G. P. and Stouten, P. F. (2000). Fast prediction and visualization of protein binding pockets with pass. Journal of computer-aided molecular design, 14(4):383-401.

Chaires, J. B. (2008). Calorimetry and thermodynamics in drug design. Annu. Rev. Biophys., 37:135-151.

Chawla, N. V., Bowyer, K. W., Hall, L. O., and Kegelmeyer, W. P. (2002). Smote: synthetic minority over-sampling technique. Journal of artificial intelligence research, 16:321-357.

Chi, P.-H., Scott, G., and Shyu, C.-R. (2005). A fast protein structure retrieval system using image-based distance matrices and multidimensional index. International Journal of Software Engineering and Knowledge Engineering, 15(03):527-545.

Chothia, C. and Lesk, A. M. (1986). The relation between the divergence of sequence and structure in proteins. The EMBO journal, 5(4):823-826.

Cortes, C. and Vapnik, V. (1995). Support-vector networks. Machine Learning, 20(3):273-297.

Dunn, D. and Higgins, W. E. (1995). Optimal gabor filters for texture segmentation. IEEE Transactions on image processing, 4(7):947-964.

Fox, N. K., Brenner, S. E., and Chandonia, J.-M. (2013). Scope: Structural classification of proteins-extended, integrating scop and astral data and classification of new structures. Nucleic acids research, 42(D1):D304-D309.

Freund, Y. and Schapire, R. E. (1997). A decision-theoretic generalization of on-line learning and an application to boosting. Journal of computer and system sciences, 55(1):119-139.

Ho, T. K. (1995). Random decision forests. In Proceedings of 3rd international conference on document analysis and recognition, volume 1, pages 278-282. IEEE.

Holm, L. and Sander, C. (1993). Protein structure comparison by alignment of distance matrices. Journal of molecular biology, 233(1):123-138.

Holm, L. and Sander, C. (1997). Dali/fssp classification of three-dimensional protein folds. Nucleic acids research, 25(1):231-234.

Jain, A. K. and Bhattacharjee, S. (1992). Text segmentation using gabor filters for automatic document processing. Machine vision and applications, 5(3):169-184.

Jain, A. K. and Farrokhnia, F. (1991). Unsupervised texture segmentation using gabor filters. Pattern recognition, 24(12):1167-1186.

Jain, A. K., Ratha, N. K., and Lakshmanan, S. (1997). Object detection using gabor filters. Pattern recognition, 30(2):295-309.

Karim, R., Aziz, M. M. A., Shatabda, S., Rahman, M. S., Mia, M. A. K., Zaman, F., and Rakin, S. (2015). Comograd and phog: From computer vision to fast and accurate protein tertiary structure retrieval. Scientific Reports, 5:13275 EP -. Article.

Kohavi, R. (1995). A study of cross-validation and bootstrap for accuracy estimation and model selection. In Ijcai, volume 14, pages 1137-1145. Montreal, Canada.

Lowe, D. G. (2004). Distinctive image features from scale-invariant keypoints. International journal of computer vision, 60(2):91-110.

Maron, M. E. (1961). Automatic indexing: An experimental inquiry. J. ACM, 8(3):404-417.

Mehrotra, R., Namuduri, K. R., and Ranganathan, N. (1992). Gabor filter-based edge detection. Pattern recognition, 25(12):1479-1494.

Ojala, T., Pietikainen, M., and Harwood, D. (1994). Performance evaluation of texture measures with classification based on kullback discrimination of distributions. In Pattern Recognition, 1994. Vol. 1-Conference A: Computer Vision \& Image Processing., Proceedings of the 12th IAPR International Conference on, volume 1, pages 582-585. IEEE.

Ojala, T., Pietikainen, M., and Maenpaa, T. (2002). Multiresolution gray-scale and rotation invariant texture classification with local binary patterns. IEEE Transactions on pattern analysis and machine intelligence, 24(7):971-987.

Patching, S. G. (2014). Surface plasmon resonance spectroscopy for characterisation of membrane protein-ligand interactions and its potential for drug discovery. Biochimica et Biophysica Acta (BBA)Biomembranes, 1838(1):43-55. 
Rossi, A. M. and Taylor, C. W. (2011). Analysis of protein-ligand interactions by fluorescence polarization. Nature protocols, 6(3):365.

Shyu, C.-R., Chi, P.-H., Scott, G., and Xu, D. (2004). Proteindbs: a real-time retrieval system for protein structure comparison. Nucleic Acids Research, 32(suppl_2):W572-W575.

Singh, A. P. and Brutlag, D. L. (1997). Hierarchical protein structure superposition using both secondary structure and atomic representations. In Ismb, volume 5, pages 284-293.

Sousa, S. F., Ribeiro, A. J., Coimbra, J., Neves, R., Martins, S., Moorthy, N., Fernandes, P., and Ramos, M. (2013). Protein-ligand docking in the new millennium-a retrospective of 10 years in the field. Current medicinal chemistry, 20(18):2296-2314.

Srivastava, S., Lal, S. B., Mishra, D., Angadi, U., Chaturvedi, K., Rai, S. N., and Rai, A. (2016). An efficient algorithm for protein structure comparison using elastic shape analysis. Algorithms for Molecular Biology, 11(1):27.

Steinbrecher, T. and Labahn, A. (2010). Towards accurate free energy calculations in ligand proteinbinding studies. Current medicinal chemistry, 17(8):767-785.

TAYLOR, W. R. (1999). Protein structure comparison using iterated double dynamic programming. Protein Science, 8(3):654-665. 